\title{
DA FUNDAMENTAÇÃO ONTOLÓGICA À REPRESENTAÇÃO DE PROCESSOS CLÍNICOS: UMA ABORDAGEM TEÓRICA
}

\author{
FROM ONTOLOGICAL FOUNDATION TO THE \\ REPRESENTATION OF CLINICAL PROCESSES: A \\ THEORETICAL APPROACH
}

\author{
Elisângela Cristina Aganettea \\ Livia Marangon Duffles Teixeirab
}

\begin{abstract}
RESUMO
Introdução: Gerenciar problemas relativos à organização da informação independente da área de conhecimento é uma tarefa árdua e dispendiosa, uma vez que demandam conhecimentos específicos, metodologias apropriadas e ferramentas de suporte adequadas. Neste contexto, as ontologias e a modelagem de processos tem se destacado como soluções para gestão de tais problemas, aliadas, são uma alternativa para alcançar a interoperabilidade entre sistemas. Objetivo: Diante disso, o presente artigo busca apresentar uma fundamentação filosófica, ontológica e em gestão relativa a processos, além de evidenciar que os respectivos fundamentos são capazes de sustentar a necessidade de melhorias em modelagem de processos de negócios. Metodologia: Para tal, apresenta-se uma contextualização teórica sobre processos no âmbito filosófico, em gestão corporativa, em ontologias de domínio e finalmente, contextualiza o corpo teórico em gestão de processos clínicos. Resultados: Como resultado, o artigo evidencia o avanço do entendimento dos processos em diferentes âmbitos de aplicação. Com o avançar da pesquisa, espera-se que seja possível identificar nos processos em modelagem as suas características essenciais e sua adequada categorização. Assim, por meio das notações, os processos apresentarão subsídios para a representação da realidade e das diferentes entidades processuais a eles relacionadas. Esses subsídios poderão se configurar como uma metodologia de referência para a comparação de processos e validação do modelo. Conclusões: Os processos clínicos, objetivo final da pesquisa em andamento, se desenvolvem no contexto da saúde e se referem à rotina em clínicas, ambulatórios, hospitais ou até mesmo atividades administrativas. Esses processos são caracterizados pelo envolvimento com prestação de cuidados envolvendo vidas, onde o tempo é algo precioso para tomada de decisão. Espera-se que os desdobramentos proporcionados
\end{abstract}

a Doutora em Ciência da Informação pela Universidade Federal de Minas Gerais (UFMG). Professora do Departamento de Organização e Tratamento da Informação da Universidade Federal de Minas Gerais (UFMG). E-mail: elisangelaaganette@gmail.com.

b Doutora em Gestão \& Organização do Conhecimento pela Universidade Federal de Minas Gerais (UFMG). E-mail: liviamarangon@gmail.com. 
por este estudo sejam utilizados como fundamentação teórica para a utilização de ontologias orientadas a processos no ambiente clínico, contribuindo para o seu desenvolvimento no campo da Ciência da Informação.

Descritores: Ontologias. Gestão de Processos de Negócios. Modelagem de Processos. Representação da Informação.

\section{INTRODUÇÃO}

Tratar os problemas relativos à organização da informação independente da área de conhecimento é uma tarefa árdua e na maioria das vezes complexa e dispendiosa. Essas iniciativas demandam conhecimentos específicos, metodologias apropriadas e ferramentas de suporte adequadas para tratar tais problemas. Representar as informações de maneira eficaz se configura uma das atividades necessárias para se alcançar a tão almejada representação e organização da informação de um determinado domínio do conhecimento. A representação da informação consiste em um processo complexo e necessita, na maioria das vezes, de habilidades intelectuais específicas. Ela envolve tanto a análise quanto a reprodução de conceitos e significados construídos pela mente humana em algum suporte, como em um dispositivo eletrônico ou no papel, por meio de símbolos e linguagens. Teóricos de diferentes áreas do conhecimento corroboram com a afirmativa da complexidade em se representar a informação, tais como: Lwoff (1970), Kobashi e Tálamo (2003), Francelin e Pellegatti (2004), Pinheiro (1999), Alvarenga Neto (2008). Mendes (1998, p.2) afirma explicitamente que "[...] capturar o conhecimento humano e torná-lo explícito não é uma tarefa simples." e Campos (2004, p. 24) corrobora com tal afirmativa pontuando que "A representação perfeita de um determinado objeto é, em geral, impossível. A única representação completamente precisa de um objeto é o objeto em si mesmo".

Uma ontologia, de acordo com Gruber (1993), é uma especificação explícita e formal de uma conceitualização compartilhada. No contexto organizacional, Uschold et al. (1997) apresentam as ontologias por suas possibilidades de organização, manipulação e representação de informações nas organizações, por meio de termos referentes à entidades do mundo real e das suas relações entre si. Neste mesmo contexto, no que se refere a um 
processo de negócio, este representa uma sequência lógica de atividades a serem executadas dentro de organizações para a entrega de resultados aos clientes (VERNADAT, 1996). Busca-se assim, uma orientação voltada para processos que garanta a eficiência organizacional, a rapidez e a eficácia das atividades de trabalho, focada em atividades geradoras de valor para os clientes e que questiona de alguma maneira, as atividades que dificultem alcançar os objetivos. A orientação por processos, com o foco nas atividades geradoras de valor para os clientes e a percepção da organização como uma cadeia de atividades interligadas por departamentos, trouxe várias respostas para muitos problemas que as organizações enfrentam, por terem suas estruturas gerenciais organizadas por função e orientadas para produtos (GARVIN, 1998).

No que tange aos "processos clínicos", domínio temático que trataremos no presente artigo, cabe ressaltar que são processos de negócios que se desenvolvem no contexto de atendimento da área da saúde, ou seja, em clínicas, ambulatórios e hospitais. Dessa maneira, envolvem vidas e tempo, sendo este último precioso e que deve ser considerado, independe do modo que as informações são gerenciadas e disponibilizadas. Pela evolução dos recursos tecnológicos, já se esperava que em algum momento o papel fosse substituído. A conversão pelo meio eletrônico de modo gradativo, no que diz respeito ao conceito de pesquisa e acesso imediato, passa a ser realidade também na área médica. O problema é que, nem sempre, este acesso acontece de modo simples, fácil e intuitivo, uma vez que não existe um instrumento de representação que intermedeie o usuário e a base de dados (AGANETTE, 2015). Fato é que, gerir os processos de negócio, representar as informações geradas e garantir o acesso às mesmas neste contexto, se configura uma adequação às demandas de um mercado que exige maior eficiência e eficácia nas atividades do negócio para a tomada de decisão, além de viabilizar maior controle e gestão pela administração.

Diante de tal cenário, fica evidente que um único instrumento não é suficiente para tal demanda. Faz-se necessário a correlação de duas soluções agregadoras e complementares. Gruninger e Pinto (1995), Uschold et al. (1997), Haller, Gaaloul e Marmolowski (2008) associam teoricamente a modelagem de 
processos e as ontologias, sugerindo a criação de ontologias a partir de modelos de processos. Os ganhos são tangíveis nessa união, uma vez que as ontologias podem, de forma prática, representar graficamente um recorte do mundo real, prover soluções semânticas, propiciar e melhorar a interoperabilidade entre sistemas e automatizar processos de inferências. Segundo Smith (2004), as ontologias vêm exercendo um papel de extrema importância e notoriedade, principalmente na área médica, uma vez que propõem iniciativas de padronização da linguagem, função semelhante àquela desempenhada pelas terminologias clínicas, como a CID (Classificação Internacional de Doenças) e a SNOMED CT (Systematized Nomenclature of Medicine Clinical Terms).

As ontologias surgem com propostas e iniciativas de padronização da linguagem e são usadas tanto para auxiliar na recuperação de informação como no processamento dos registros eletrônicos dos pacientes (AGANETTE, 2015). Já a modelagem de processos se apresenta como uma solução, por meio da qual se é possível evidenciar hiatos, planejar correções e melhorias, implantar novas e melhores formas de execução e automatizar atividades e processos documentais, presentes em abundância nesse ambiente e ainda, gerenciar o conhecimento corporativo. Pode-se afirmar assim que, a representação de processos de forma bem fundamentada e apresentando maior semelhança com a realidade representada, possibilitará a automação de fluxos documentais e proverá a interoperabilidade com outros sistemas de informações, sistemas legados e banco de dados. Assim, resultará em maior facilidade para automatização dos processos mapeados e melhor experiência de todos os tipos de usuários que podem se beneficiar com a implantação de projetos desta natureza. Para melhor entendimento das temáticas, serão brevemente apresentadas, nas seções seguintes, algumas dessas abordagens.

Sendo assim, o objetivo aqui delimitado é apresentar uma consistente fundamentação filosófica, ontológica e em gestão relativa a processos, além de evidenciar que os seus respectivos fundamentos abordados são capazes de sustentar a necessidade de melhorias em modelagem de processos de negócios. Para tal, apresenta-se uma contextualização teórica sobre processos no âmbito filosófico, em gestão corporativa (com ênfase em Business Process 
Management - BPM e Business Process Management Notation - BPMN), nas ontologias de domínio, sendo estas a Basic Formal Ontology (BFO) e Unified Foundational Ontology (UFO) e finalmente, contextualiza o corpo teórico em gestão de processos clínicos. Entende-se a necessidade de conhecer diferentes abordagens sobre processos, suas definições, suas características e seus tipos, ou seja, a sua essência, para que seja possível avaliar e considerar melhorias no ato de modelagem e representação de seus artefatos.

Compreende-se que a análise ontológica de processos seja capaz de oferecer subsídios que fundamentam percepções baseadas na realidade do contexto. Com intuito de se contribuir para a qualificação científica dos estudos, utilizou-se da pesquisa bibliográfica, como procedimento metodológico, por meio da fundamentação conceitual, que consistiu em apresentar o marco teórico que norteia todo o desenvolvimento do trabalho. Outrossim, trata-se de um estudo teórico que objetiva descrever e apresentar a pesquisa. Vale ressaltar que a pesquisa teórica não implica em imediata intervenção na realidade, mas nem por isso deixa de ser importante. O seu papel é decisivo na criação de condições para a intervenção, uma vez que "O conhecimento teórico adequado acarreta rigor conceitual, análise acurada, desempenho lógico, argumentação diversificada, capacidade explicativa" (DEMO, 1994, p. 36).

Na perspectiva deste artigo, pretendeu-se contribuir para a qualificação dos estudos teóricos que estabelecem a pesquisa bibliográfica como principal procedimento metodológico, por meio das seguintes etapas: i) inicialmente escolheu-se os procedimentos e parâmetros que permitiram realizar a pesquisa pelos conteúdos, assim como classificar o material e o conteúdo resultante; ii) em seguida definiu-se o percurso de pesquisa realizado, direcionado às formas de apresentar e de analisar os conhecimentos obtidos; iii) como análise dos conhecimentos, houve a possibilidade de apontar a possibilidade de um direcionamento prático, que ainda deverá ser submetido a desenvolvimento e validação e por fim; v) realizaram-se as configurações finais.

Após esta introdução, o presente artigo apresenta as seguintes seções: em (2), proporcionam-se teorias, conceitos e características referente a processos na filosofia e na área de gestão; em (3) apontam-se algumas 
discussões e considerações finais.

\section{PROCESSOS: TEORIAS E CONCEITOS}

\subsection{Processos NA fiLosofia}

O termo "processo" é nativo do latim processus, que indica movimento de avanço sequencial e sugere uma mudança em um percurso definido. Em 1978, Mourelatos (filósofo linguista) propõe uma classificação dos tipos verbais para viabilizar os estudos sobre processos, tanto nos âmbitos da filosofia da ação, na filosofia da mente, nas ontologias, na linguística e também na história da filosofia (MOURELATOS, 1978). O autor categoriza os verbos entre estados e ocorrências. O estado denota não ações que não se desenvolvem no tempo. São exemplos os verbos desejar, querer, amar, odiar, dominar e as sentenças "Ana sabe a resposta" e "Você parece muito com sua irmã" (VENDLER, 1967). Já as ocorrências se dividem entre processos e eventos. Para o linguista, o que diferencia os processos dos eventos é a semelhança entre as partes que compõem um processo. Ou seja, para Mourelatos (1978) os processos ocorrem de forma homogênea, de forma que todas as partes de um processo são equivalentes às outras demais. Por exemplo, estar andando implica "ter andado". Outrossim, os processos são identificados como tipos de ocorrências. Já Stout (1997), vai além do pensamento de Mourelatos (1978) de que os processos ocorrem de forma homogênea. De acordo com Stout (1997), se ao interromper um processo e as partes resultantes não forem idênticas ao processo como um todo, indica a presença de mais de uma fase. Assim, cada fase pode ser pensada como estado de coisas, eventos ou sub-processos.

O fio condutor de Stout (1997) é baseado em processos persistindo no tempo, uma vez que a pergunta "what is happening?" está continuamente presente na sua totalidade em diferentes momentos, caracterizando como entidades ocorrentes. Concluindo seu pensamento, Stout (1997, p. 26, tradução nossa) diferencia os processos dos eventos "[...] uma vez que os eventos estendem mas não

\footnotetext{
c "O que está acontecendo?"
} 
persistem no tempo e os processos persistem mas não estendem no tempo, processos nunca são eventos". Já Henning (2008) foca seu estudo diferenciando as entidades ocorrentes dos demais tipos de entidades temporais. De acordo com o autor, as entidades temporais são aquelas que "acontecem" ou "ocorrem" e as que ocorrem são as entidades denominadas ocorrentes. A taxonomia das entidades temporais "ocorrentes" pode ser representada conforme Figura 1 a seguir:

\section{Figura 1 - Taxonomia de entidades temporais}

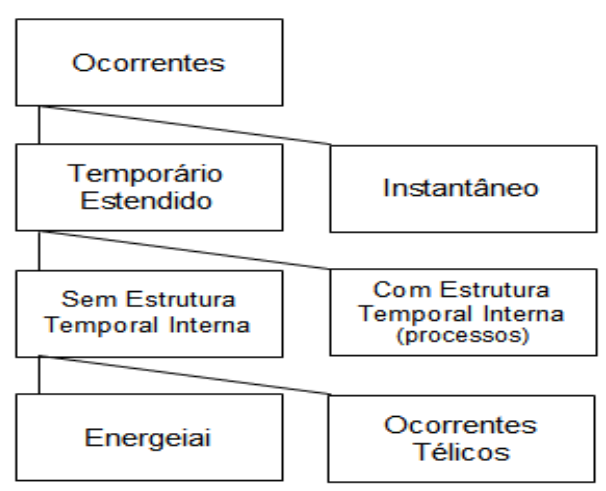

Fonte: adaptado de Henning (2008, p. 265).

Henning (2008) definiu processos enquanto ocorrentes internamente estruturados e apresentou dois níveis de estruturação que um ocorrente pode ter. O primeiro nível é quando se sabe apenas as circunstâncias sob as quais o ocorrente começa e termina. Por exemplo, procurar um objeto perdido não tem estrutura temporal interna e não há como medir o quanto já foi concluído. $O$ segundo nível é quando o ocorrente já possui estrutura típica e é possível medir sua realização. Por exemplo, a realização de um exame de imagem. Nesse nível são encontrados os ocorrentes télicos e os ocorrentes energeiai. Os ocorrentes estruturados são denominados télicos no sentido de haver um fim previsto no processo (pelo estado de estar completo ou terminado) e pela possibilidade de sua interrupção antes da conclusão. Já os energeiai se referem aos ocorrentes que não podem ser interrompidos antes de estarem completos.

Apesar das diferenças mais sutis nas abordagens, os três autores identificam e reconhecem o processo enquanto uma entidade ocorrente. Uma característica de uma entidade ocorrente é a sua relação de dependência de 
uma entidade continuante para sua existência. A relação de dependência é denominada participação (ALMEIDA, 2016), que é explicada pela atuação de uma substância em um processo, ou ainda, pode-se afirmar que processos dependem de objetos e estes desempenham funções.

\subsection{Processos ENQUANTO CATEGorias ONTOLÓgICAS}

Na literatura da filosofia, como demonstrado por Mourelatos (1978), Stout (1997) e Henning (2008), pode-se perceber diferença conceitual para as "entidades localizadas no tempo". Já Smith (2003) e Guizzardi, Falbo e Guizzardi (2008) percebem essa diferença por meio do ponto de vista ontológico. Para Smith (2003), trata-se de um "compromisso ontológico" evidenciar as diferenças entre as entidades, sejam estas objetos e/ou processos, que se tenta representar. As ontologias de alto nível são aquelas que identificam as categorias mais fundamentais de entidades do mundo, ditas universais. Para a representação de um domínio do conhecimento elas diferenciam suas entidades participantes entre objetos e processos. A BFO (Basic Formal Ontology) e a UFO (Unified Foundational Ontology) são exemplos representativos de ontologias de alto nível, cuja noção de processos são equivalentes, embora existam especificidades que as diferenciam - o que justifica suas escolhas no estudo. As ontologias de alto nível são utilizadas em diferentes áreas do conhecimento, como na medicina, em engenharia, na economia e outros. Elas apoiam o desenvolvimento de uma estrutura de universais e classes propiciando a sua reutilização e a interoperabilidade entre sistemas. Almeida (2013) esclarece que a diferença entre classes e universais consiste em: enquanto universais são entidades independentes da vontade humana, classes são criações da mente para fins específicos. Como o interesse de pesquisa está em processo, este será explorado brevemente a seguir sob as referidas óticas.

\subsection{BFO: OCORRENTE TEMPORARIAMENTE ESTENDIDO COM ESTRUTURA TEMPORAL INTERNA}

A BFO é uma ontologia formal que fornece suporte ao desenvolvimento das ontologias de domínio. O principal uso da BFO quanto ao conteúdo 
semântico, refere-se à utilização de seus universais na descrição do conhecimento de entidades, sendo que estas não são as únicas entidades usadas para descrever partes da realidade do mundo. As entidades são os elementos utilizados para referenciar objetos do mundo real e as representadas na BFO são aquelas estudadas pela ciência empírica e que afetam ou estão envolvidas em atividades humanas, tais como o processamento de dados, planejamento e organização (SMITH et al., 2012). Por meio das relações ontológicas formais (is_a e part-of) e das instâncias, representam-se casos reais de um universal correspondente (MENDONÇA, 2015). Na BFO as entidades estão agrupadas em dois grandes grupos distintos (GRENON; SMITH; GOLDBERG, 2004): i) continuant (continuantes) - entidades que persistem ao longo do tempo mantendo sua identidade e que não possuem partes temporais; ii) occurrent (ocorrentes) - entidades que se revelam, se manifestam ou se desenvolvem ao longo do tempo e possuem partes temporais.

Ainda segundo Grenon, Smith e Goldberg (2004), a divisão da BFO em duas perspectivas diferentes demandou a criação de duas ontologias distintas na sua formação, sendo elas: i) Ontologia SNAP - que agrupa as entidades continuantes e; ii) Ontologia SPAN - que agrupa as entidades ocorrentes, como pode ser visto na figura 2 abaixo.

Figura 2 - Localização da entidade processo na BFO SNAP

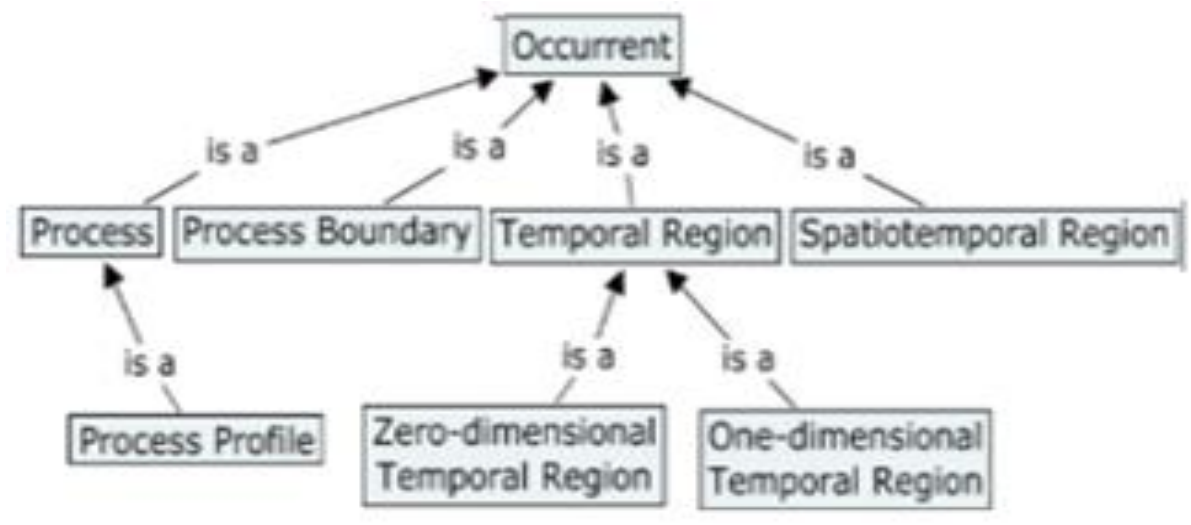

Fonte: adaptado de Mendonça (2015, p. 99)

Henning (2008) busca evidenciar as origens aristotélicas dos estudos sobre processo e o define como um "ocorrente internamente estruturado, temporariamente estendido" (HENNING, 2008, p. 269, tradução nossa), 
conforme já apresentado na seção anterior. Os demais ocorrentes, sejam instantâneos ou até mesmo os temporariamente estendidos sem estrutura temporal interna, não podem ser considerados processos. A diferença, de acordo com o autor, consiste na possibilidade de determinar o quanto do ocorrente já aconteceu, enquanto ele continua ocorrendo, quando existe estrutura temporal interna em uma ordem típica.

A principal característica de um ocorrente temporariamente estendido complexo é a sua capacidade de ser subdividido em outros ocorrentes temporariamente estendidos mais específicos. Em contrapartida, essa característica não se aplica aos ocorrentes simples. No caso de processos, ou seja, de um ocorrente temporariamente estendido e internamente estruturado, trata-se de um processo simples e este não deve ser composto de nenhum outro processo e ao mesmo tempo este deve ser capaz de comunicar o quanto está completo durante a sua execução. E ao decompor um processo complexo em partes temporariamente estendidas, culminará em processos simples (HENNING, 2008).

\subsection{UFO: ENTIDADE PERDURANTE}

A UFO é uma ontologia de fundamentação desenvolvida com o objetivo de prover uma teoria para resolver problemas clássicos de modelagem conceitual. A UFO inclui teorias sobre as partes e o todo, tipos, estruturas taxonômicas, relacionamentos, atributos e atributos de espaço, papéis e qualidades individuais, além de outras coisas (MENDONÇA, 2015). Assim como na BFO, as entidades são diferenciadas, de acordo Guizzardi, Falbo e Guizzardi (2008): i) eventos ou ocorrências - acontecem no tempo no sentido de se estenderem no tempo, acumulando partes temporais; ii) objetos - estão inteiramente presentes em qualquer instante de tempo em que estiverem presentes; e iii) entidades sociais - tanto objetos quanto eventos, distinção não pertinente no momento. Sob essa perspectiva, a UFO foi subdividida em três fragmentos: i) UFO-A - objetos e outros endurantes; ii) UFO-B - eventos e outros perdurantes; e iii) UFO-C - que define termos relacionados às esferas sociais $\mathrm{e}$ aspectos linguísticos. Deste modo, os eventos são diferenciados por eventos 
complexos e eventos atômicos. A figura 3, a seguir, apresenta um recorte da UFO-B, como exemplo da categoria de perdurantes:

Figura 3 - Recorte da UFO-B

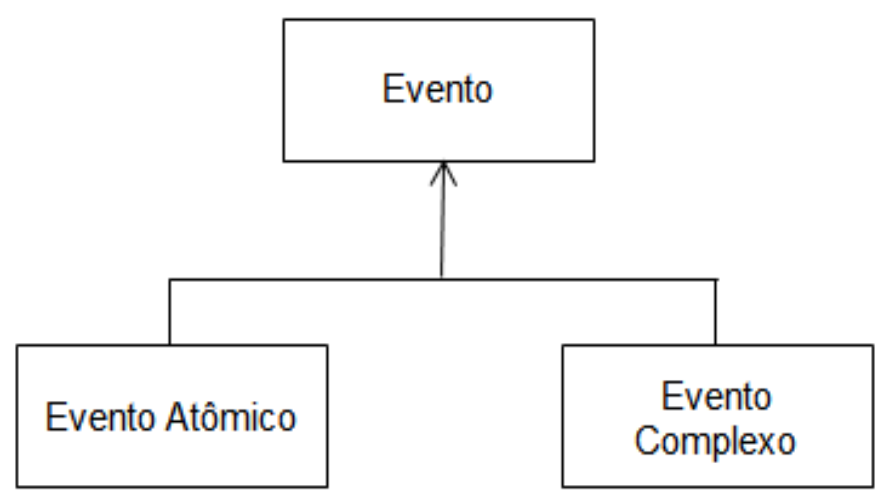

Fonte: adaptado de Guizzard e Wagner (2004, p.11).

Guizzard e Wagner (2010) caracterizam os eventos como entidades dependentes dos endurantes para existirem e que podem também ser decompostas em partes temporais, como na BFO.

Os autores explicam que os eventos atômicos são aqueles que não podem ser decompostos em partes (por exemplo, o bloqueio na rede em um jogo de voleibol), enquanto os eventos complexos são constituídos por outros eventos (por exemplo, um ataque em um jogo de voleibol, constituído pelos eventos passar a bola para o jogador levantador, este posicionar a bola no local e altura adequada e outro jogador realizar o movimento de atacar a bola para 0 adversário) que podem ser atômicos ou complexos.

Os eventos complexos são os também denominados processos, conforme figura 4. Um processo é uma "sequência de duas ou mais ocorrências, possivelmente paralelas, de eventos atômicos" (GUIZZARD; WAGNER, 2005, p. 359, tradução nossa).

Os eventos são entidades dependentes que são caracterizados também pela dependência de seus participantes. Estes podem ser pessoas ou objetos (como os documentos) que são representados pelo tipo Participação (Participation), que também podem ser identificados como eventos atômicos ou eventos complexos, constituintes do processo. 
Figura 4 - Localização e entidades dos processos

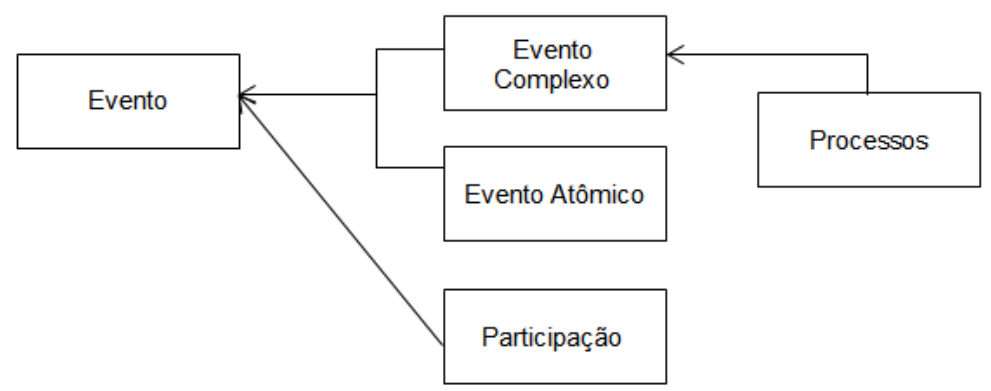

Fonte: adaptado de Guizzard e Wagner (2010, p. 657) e Rocha (2012, p. 73)

Pode-se destacar na BFO que, o que tipifica o processo como tal, é a sua estrutura temporal interna, possibilitando determinar o quanto do ocorrente já aconteceu enquanto ele continua ocorrendo. Já na UFO, a sua tipificação vem pela sequência de ocorrências que o compõe. Considera-se a diferença entre os tipos apresentados na perspectiva filosófica que fundamenta cada uma delas. Campos e Campos (2012), realizaram uma análise comparativa das características da UFO e da BFO, evidenciando-se assim o que veio ao encontro dos nossos propósitos. Segundo as autoras, a UFO possui um modelo mais detalhado e mais flexível para modelar, como, por exemplo, o da qualidade e o do encadeamento de processos no tempo. No entanto assume o compromisso com a existência de entidades abstratas, que não possuem correspondentes no mundo real. Já a BFO apresenta um modelo menos detalhado, porém aderente com as entidades do mundo real, adequado para as necessidades da comunidade biomédica.

\subsection{PROCESSOS ENQUANTO DISCIPLINA DE GESTÃo}

Um processo do ponto de vista da gestão, é um conjunto sequencial e peculiar de ações que objetivam atingir determinada meta. Ele viabiliza criar, inventar, projetar, transformar, produzir, controlar, manter e usar produtos ou sistemas. As definições de áreas gerenciais basicamente se atém a uma visão comum, em que o processo é algo que ocorre entre uma entrada e uma saída.

Para Hammer e Champy (1994), um processo consiste em um grupo de atividades realizadas numa sequência lógica com o objetivo de produzir um bem ou um serviço que tem valor para um grupo específico de clientes. Harrington, 
Esseling e Nimwegen (1997) afirmam que o processo é um fluxo de trabalho com entradas (input) e saídas (output) bem definidas e tarefas discretas, seguindo uma sucessão clara onde umas dependem das outras. Já para Cruz (2015), o processo é um conjunto de elementos que guia o indivíduo ou um grupo entre o início e o fim de um trabalho específico, de forma que o que deve ser feito seja feito sem desvios, alcançando a meta ou objetivo.

Existem diferentes tipos de metodologias para a representação dos processos, como fluxogramas, UML (Unified Modeling Language) e BPMN (Business Process Model and Notation), que se estabelecem na disciplina da gestão de processos de negócios (BPM - Business Process Management).

\subsection{GESTÃo de PROCESSOS DE NEGócIOS Vs. GESTÃo POR PROCESSOS DE NEGÓcIO}

A gestão de processos de negócios é uma área do conhecimento que, empírica e teoricamente, objetiva conhecer para entender as atividades que ocorrem em uma organização, com propósitos que vão desde a otimização da execução das respectivas atividades à automação e implantação destas. Para Recker et al. (2006) apud Aagesen e Krogstie (2013), a BPM pode ser descrita como uma

Forma estruturada, coerente e consistente de compreender, documentar, modelar, analisar, simular, executar e continuamente mudar processos de negócio, de ponta a ponta e todos os recursos envolvidos, com base em sua contribuição para o desempenho dos negócios (RECKER et al., 2006, p. 2, traduzido por AAGESEN; KROGSTIE, 2013, p. 126).

Segundo o Guia BPM CBOK (BPMP, 2009) a gestão de processos de negócio (ou BPM) é uma abordagem disciplinada para identificar, desenhar ou projetar, executar, medir, monitorar e controlar processos de negócio, automatizados ou não, para alcançar consistência e resultados alinhados aos objetivos estratégicos da organização. Ela envolve ainda, com ajuda de tecnologia, formas de agregar valor, melhorias, inovações e o gerenciamento dos processos de ponta a ponta, levando a uma melhoria do desempenho organizacional e dos resultados de negócios.

Já a gestão POR processos consiste na efetiva e real integração e na relação entre os processos e respectivas atividades realizadas em uma 
instituição por seus diferentes setores. A gestão por processos visa 0 fortalecimento da visão sistêmica institucional, permitindo assim, uma visão holística de todo o cenário. Ressalta-se que as instituições são organismos vivos e seu bom funcionamento depende da boa relação entre as pessoas e as atividades, ou seja, estes devem atuar de modo interdependente. Em suma, a gestão por processos permite que as equipes sejam envolvidas em um ambiente cooperativo e de compartilhamento de informações (FNQ, 2007).

Segundo a FNQ (2007) é primordial que se valorize o processo de mapeamento dos processos da organização. Sem um mapeamento bem feito não existe a "gestão por processos". O mapeamento deve ser feito com o mais absoluto detalhamento e cuidado, sendo considerado como a ferramenta balizadora de decisões rotineiras acerca de mudanças operacionais. Além disso, deve ser utilizado como alicerce para promover o compartilhamento de informações e a responsabilidade pelas atividades rotineiras, para assim, finalmente, se colher os frutos de uma administração efetivamente voltada a processos.

A representação dos processos de negócios é realizada por meio da modelagem desses processos, que são identificados por diagramas que se utilizam de notações, como por exemplo, a Business Process Model and Notation (BPMN). A BPMN é uma notação para a modelagem de processos organizacionais onde os modelos de processos gerados podem ser utilizados para diferentes fins, conforme aponta Krogstie, Dalberg e Jensen (2008): i) criação de sentido e comunicação humana; ii) análise assistida por computador; iii) gestão da qualidade; iv) implementação e ativação do modelo, para integrálo em um sistema de informação; v) utilização do modelo como contexto para um projeto de desenvolvimento de sistema, entre outros.

Em 2004 a BPMN foi apresentada pela Object Management Group (OMG) como a linguagem padrão para a atividade de modelagem e, desde então, tem sido avaliada pela comunidade acadêmica levando ao entendimento de que, sua adequação, melhoria e atualização são amplamente contempladas. O OMG é um consórcio de padrões tecnológicos sem fins lucrativos, cujo objetivo consiste em oferecer uma linguagem padrão, comum, aceita e compreendida por toda a 
comunidade envolvida na atividade de modelagem de processos. Por meio dos seus construtos representam-se as atividades que acontecem em determinado ambiente para um determinado objetivo, culminando em um modelo que será utilizado para diferentes fins.

O contexto da área da saúde, assim como de outras áreas, como por exemplo, comércio varejista ou a área de geração e distribuição de energia, apresentam necessidades e demandas para se manterem competitivas no mercado. Instituições como hospitais ou clínicas precisam gerir melhor seus recursos além de garantir a qualidade de seus procedimentos e minimizar a quantidade de erros por meio da institucionalização de padrões e de procedimentos. Por isso, demandam integração de informações, tanto administrativas quanto relativas aos cuidados da saúde de um paciente. As demandas por melhorias em gestão de informação advindas de instituições da área de saúde apresentam alguns aspectos comuns, entre eles: i) hospitais possuem uma estrutura de funcionamento complexa, o que dificulta 0 alinhamento entre a gestão de processos e as tecnologias, culminando em um acúmulo de tecnologias diversas aplicadas em tarefas isoladas ou fragmentos de processos; ii) melhoria do padrão e da expectativa de vida proporcionada pelos tratamentos médicos, que resultam em um aumento dos custos com os cuidados da saúde; iii) crescente pressão pelo controle dos custos associados aos tratamentos garantindo a eficiência, a segurança e a qualidade dos mesmos; iv) utilização de prontuários eletrônicos e por fim, v) uma tendência pelo uso de sistemas centrados nos cuidados dos pacientes (RECTOR, 1999).

A ausência ou o mau uso das tecnologias em hospitais e instituições de saúde geralmente resulta em problemas, por exemplo, um maior tempo de espera dos pacientes por atendimento médico, procedimentos médicos difíceis de serem executadas, perda de resultados de exames, repetições e custos excessivos que dificultam a otimização de processos (MARTINHO; RIJO; NUNES, 2015, p. 1227). Os autores ainda consideram que a área da saúde anseia por adoções eficientes em gestão de processos, uma vez que na realidade, observam-se diferentes tipos de tecnologias (nem sempre interoperáveis) demandando um monitoramento cada vez maior para um melhor 
uso de recursos e maior satisfação de usuários. Shitkowa, Taratukhin e Becker (2015) explicam que os hospitais e a área da saúde estão atualmente passando por um momento onde necessitam alinhar seus processos administrativos e suas atividades de cuidados médicos para aumentar sua eficiência e melhorar a qualidade dos seus serviços. Esse alinhamento é obtido por meio dos Protocolos Clínicos (CP - em inglês, Clinical Pathways), que são instrumentos internacionalmente aceitos e que podem ser definidos como "um método para a gestão do cuidado do paciente de um grupo bem definido de pacientes durante um período de tempo bem definido" (SHITKOWA; TARATUKHIN; BECKER, 2015, p. 206, tradução nossa).

\subsection{BPMN EM PROCESSOS CLÍNICOS}

Braum et al. (2015, p. 839) indicam a BPMN como a notação mais promissora em relação às demais para modelar os chamados protocolos clínicos $^{d}$, indicando ainda diferentes razões para tal, a saber: i) configurar-se como padrão oficial da ISO e atingir uma notável prevalência e aceitação tanto na academia como na indústria; ii) fornecer um metamodelo bem definido, iii) suportar a derivação de modelos de workflow interpretável por computador e estar incorporado em uma estrutura orientada para a execução; iv) fornecer um grande conjunto de conceitos genéricos de modelagem de processos que implica em grande expressividade; v) fornecer um mecanismo de extensão leve e explicitamente definido para extensão e adaptação de domínio específico; e vi) e existência de vários trabalhos de pesquisa sobre BPMN no contexto de cuidados de saúde que indicam um certo nível de proeminência dentro do referido domínio.

Simeone et al. (2013) apresentam diversas justificativas de aderência da BPMN para os processos clínicos, seja por seu consolidado padrão de formalização de representação de processos e por sua semântica explícita para

\footnotetext{
d Os protocolos clínicos são condutas e procedimentos desenvolvidos com suporte em evidências atualizadas e consistentes, que objetivam promover uma melhor prática da medicina. Esses protocolos vem auxiliar o médico em decisões sobre a melhor e mais apropriada conduta em situações clínicas específicas, permitindo resolução mais rápida e eficiente das enfermidades, gerando melhor qualidade de vida aos pacientes (CHEHUEN NETO et al., 2009).
}

Inf. Inf., Londrina, v. 24, n. 2, p. 234 - 262, maio/ago. 2019. 
execução.

A capacidade da BPMN para encapsular atividades em subprocessos também nos permite gerenciar processos complexos e reutilizar a mesma estrutura de atividades várias vezes. Ao mesmo tempo, as atividades não estruturadas ou intermediárias são representadas por meio de sub-processos ad hoc que podem ser invocados durante a simulação real de acordo com curvas probabilísticas. (SIMEONE et al., 2013, p. 63, tradução nossa).

Diferentemente a Braum et al. (2015) e Simeone et al. (2013), Shitkowa, Taratukhin e Becker (2015) apresentam uma contrapartida às linguagens de modelagens mais tradicionais, como os já citados fluxogramas, UML e BPMN, dentre outras. De acordo com os autores, essas linguagens de modelagens possuem certa deficiência em não satisfazer os requisitos de representação demandadas especificamente pelos protocolos clínicos. Eles argumentam que, se por um lado os protocolos clínicos registram um volume expressivo de informações de forma bastante detalhada, ao mesmo tempo não permitem uma análise automática, a captura e a transformação a partir de certo nível de detalhes. Eles ainda argumentam em relação à BPMN que: i) a metodologia não produz modelos intuitivos e precisa ser estendida; ii) a introdução do método está associada a um enorme investimento em tempo e mão-de-obra; e, iii) apesar ser mais eficiente do que as descrições textuais dos protocolos clínicos, é um método difícil como parte da rotina clínica diária.

Considerando que, embora seja a notação mais expressiva para a modelagem de processos de negócios e ainda assim apresenta fragilidades para o contexto clínico, é necessário pensar na agregação prática da gestão de processos à gestão de documentos e de informações. Nesse sentido, as autoras deste trabalho têm buscado tratar da importância desse agrupamento para fins de gestão e para a tomada de decisão (TEIXEIRA; AGANETTE, 2018; AGANETTE; TEIXEIRA; AGANETTE, 2018).

\subsection{GESTÃO DOS FLUXOS DE DOCUMENTOS EM PROCESSOS CLíNICOS}

Uma das principais características dos processos clínicos é a geração de documentos e informações, sejam imagens ou textos resultantes de análises clínicas que dão suporte às atividades, procedimentos e decisões tanto em nível 
operacional quanto em nível estratégico. Ressalta-se que esses documentos e informações ainda tramitam tanto no ambiente físico quanto eletrônico, tornando a gestão do fluxo ainda mais complexa. Assim, gerenciar documentos e informações deve ser, reconhecidamente, um pré-requisito para projetos de mapeamento e modelagem de processos.

Em projetos dessa natureza em que tal requisito é desconsiderado, observa-se um gap nos modelos gerados, uma vez que o documento é identificado como um artefato denominado "objeto de dados", sem contemplar sua correta e imprescindível identificação arquivística, seu fluxo e trâmite no processo ao qual está relacionado, além do seu próprio ciclo de vida. Scheuerlein et al. (2012) abordam de maneira muito superficial a vinculação dos documentos médicos aos processos de protocolos clínicos modelados em BPMN: "Na BPMN, documentos padrão, cartas médicas, relatórios de procedimentos cirúrgicos, relatórios de exames e etc., bem como documentos importantes para os checklists, podem ser vinculados diretamente à notação gráfica do protocolo clínico" (SCHEUERLEIN et al., 2012, p. 760, tradução nossa). Já Rojo, Daniel e Schrader (2012), embora contemplem em seu trabalho a interoperabilidade semântica dos registros multimídia eletrônicos de saúde de maneira específica em patologia, não abordam as tipologias documentais que compõem os respectivos registros.

Ressalta-se portanto, a necessidade e a importância da modelagem dos fluxos e trâmites documentais vinculados aos processos de negócio. Dessa forma será obtido um modelo de maturidade com nível satisfatório de gestão de documentos e informações. Aagesen e Krogstie (2015, p. 227, tradução nossa) confirmam esse entendimento ao afirmar que "Os artefatos de modelagem da BPMN são objetos de dados, grupos e anotações. Os objetos de dados não tem nenhum outro efeito sobre o processo, exceto oferecer informações sobre os recursos necessários ou produzidos pelas atividades. Os construtos de grupo são um auxílio visual usado para fins de documentação ou análise, enquanto a anotação de texto é usada para adicionar informações adicionais sobre certos aspectos do modelo". A OMG (2011) explica essa característica de modo que se justifica a aderência do desenvolvimento da pesquisa, afirmando que, embora a 
especificação ${ }^{\circ}$ da linguagem BPMN seja bastante extensa, ela especifica apenas recursos de forma abstrata como algo que pode ser referenciado por uma ou mais atividades.

\subsection{Característica essencial do processo}

O essencialismo antecede a teoria da evolução e consistiu o padrão de classificação utilizado pela taxonomia biológica. Biólogos como John Ray, Maupertuis, Bonnet, Linneaus, Buffon e Lamarck acreditavam que a maneira correta de classificar os organismos por espécie e por tipos específicos, era por meio da identificação da essência (MAYR, 1982) faltou referenciar. A principal motivação para o essencialismo consiste em explicar o comportamento dos indivíduos dentro de uma espécie. O essencialismo considera que cada entidade tem uma característica essencial, a qual é a característica que, justamente, o torna o tipo de entidade que ela é. Aristóteles denomina essa característica de "essência real" da entidade (AGANETTE, 2015).

A essência real de uma entidade ocorre em todas, mas apenas, em entidades daquele tipo específico. Para um essencialista, a essência real captura a estrutura fundamental do mundo. Para Aristóteles, uma entidade individual existe apenas como certo tipo de coisa: uma vez que deixa de ser o seu tipo, essa coisa não é mais a mesma entidade. A essência real de uma pessoa é sua racionalidade. Se tal pessoa perde essa essência, ela deixa de existir. Na teoria aristotélica, a forma, essência real, tem precedência sobre a matéria das entidades individuais (AGANETTE, 2015). Almeida (2014) explica que a característica essencial de cada espécie é justamente aquela que torna a entidade o tipo de entidade que ela é. Isso ajuda a entender porque entidades de um tipo específico são capazes de fazer o tipo de coisa que usualmente fazem, além de fornecer meios para prever e explicar seu comportamento.

No que se refere aos processos, não identificar sua característica

\footnotetext{
e A especificação distingue entre recursos humanos e não-humanos [...], o que significa que outros tipos de modelagem, como modelos de dados e informações, regras de negócios ou "modelos organizacionais e recursos" estão explicitamente fora do escopo da especificação BPMN (OMG, 2011, p. 876, tradução nossa).
} 
essencial pode gerar erros na modelagem e uma não identificação com a realidade, além de ocasionar problemas de interoperabilidade entre os sistemas utilizados. Teixeira e Aganette (2016) explicam que, para um processo ser caracterizado como um processo faz-se necessário que suas características gerais estejam explícitas. Em sequência são mapeadas as características específicas para assim definir quais dentre estas são as características "essenciais", para sua adequada modelagem e representação.

O quadro 1 pretende sintetizar as características gerais e específicas de processos, de acordo com diferentes áreas do conhecimento. É possível identificar uma ampla variedade de apontamentos, com exceção da referência às entradas/saídas do processo e que, entre o evento início e o evento fim de um processo existe uma sequência de atividades previstas com regras e objetos associados.

\section{Quadro 1 - Características de "Processo"}

\begin{tabular}{|c|c|c|}
\hline $\begin{array}{c}\text { Área de } \\
\text { conhecimento }\end{array}$ & Definição & Referência \\
\hline Filosofia & $\begin{array}{l}\text { i) entidade com limite temporal } \\
\text { ii) estrutura temporal interna }\end{array}$ & Henning (2008) \\
\hline $\begin{array}{l}\text { Gestão em Sistema } \\
\text { de Informação }\end{array}$ & $\begin{array}{l}\text { i) limites: identificam início e fim do } \\
\text { processo, caminhos e entradas e saídas } \\
\text { (informações e serviços) } \\
\text { ii) fornecedores: quem fornece as entradas } \\
\text { do processo } \\
\text { iii) clientes: quem recebe a saída do } \\
\text { processo } \\
\text { iv) integração: influência ou ligação com os } \\
\text { demais processos }\end{array}$ & $\begin{array}{l}\text { Harrington, } \\
\text { Esseling e } \\
\text { Nimwegen } \\
\text { (1997) }\end{array}$ \\
\hline $\begin{array}{l}\text { Gestão em } \\
\text { Administração }\end{array}$ & $\begin{array}{l}\text { i) objetivos do processo } \\
\text { ii) clientes do processo } \\
\text { iii) entradas físicas } \\
\text { iv) entradas lógicas } \\
\text { v) saídas físicas } \\
\text { vi) saídas lógicas } \\
\text { vii) diretrizes (regulação) } \\
\text { viii) programa de melhoria contínua } \\
\text { ix) benchmarking } \\
\text { x) metas } \\
\text { xi) alocação de recursos } \\
\text { xii) mão de obra } \\
\text { xiii) medição de desempenho } \\
\text { xiv) tecnologia de informação } \\
\text { xv) gerente do processo }\end{array}$ & Cruz (2015) \\
\hline Norma ISO & Requisitos para determinação de & ABNT NBR ISO \\
\hline
\end{tabular}




\begin{tabular}{|l|l|l|}
\hline & processos: & $9001: 2015$ \\
& i) determinar as entradas e saídas; & \\
& ii) determinar sequência e interação de & \\
& processos; & \\
& iii) determinar critérios e métodos para & \\
assegurar operação e controle dos & \\
processos; & & \\
& iv) determinar recursos; \\
& v) atribuir responsabilidades; \\
& vi) apontar riscos e oportunidades; & \\
& vii) manutenção da qualidade; \\
viii) melhoria do processo. & \\
\hline
\end{tabular}

Fonte: elaborado pelas autoras

O processo passou pela elucidação e avaliação dos seus conceitos relacionados. Na filosofia ele foi definido por Mourelatos (1978), Stout (1997) e Henning (2008), onde apesar das diferenças identificadas enquanto uma "entidade localizada no tempo", ambos reconheceram um processo como uma entidade ocorrente. Ao se verificar sob o ponto de vista ontológico, Smith (2003) e Guizzardi, Falbo e Guizzardi (2008) evidenciaram as diferenças de suas abordagens pela perspectiva das categorias ontológicas da BFO e da UFO, podendo ser objetos e/ou processos as entidades. Já no contexto da gestão, por meio de Harrington, Esseling e Nimwegen (1997), Cruz (2015) e da Norma ABNT NBR ISO 9001:2015 (específica para definição de processos), pode-se dizer que, para os autores, o processo consiste em uma sequência de ações para se alcançar uma meta. O que foi obtido nessa seção para a continuidade da pesquisa foi um avanço significativo no entendimento do conceito em diferentes abordagens, encaminhando a reflexão para a possibilidade de erros classificatórios em sistemas de recuperação de informação baseados em estruturas hierárquicas.

\section{CONSIDERAÇÕES FINAIS}

O que se pretendeu até o momento foi apresentar um estudo teórico que objetiva sustentar o desenvolvimento da pesquisa em utilização de ontologias orientadas a processos clínicos no contexto da Ciência da Informação. Entendese que a maioria das ontologias aplicadas no contexto clínico são baseadas na BFO, de fundamentação filosófica realista e portanto voltadas para a 
representação de entidades com representações no mundo real. Assim como a Ontology for General Medical Science (OGMS). A OGMS é uma ontologia de domínio que representa o processo de uma doença, desde um organismo saudável, passando pelo curso etiológico (seu agente, seus sinais e sintomas) e alcançando o plano de tratamento por meio da representação de entidades presentes em todo esse processo (doenças, suas causas, manifestações, diagnóstico e outras entidades relacionadas). Como o foco deste trabalho está direcionado aos processos de negócios clínicos e não ao processo etiológico em si, justifica-se a importância do desenvolvimento aqui apresentado.

Evidenciar o relacionamento entre processo de negócio, gestão de informações, fluxo documental e ontologias foi necessário para sustentar as propostas de melhorias em modelagem de processos clínicos. A gestão documental almejada compreende e contempla os documentos enquanto unidades de conteúdo dinâmico que possuem fluxo de aprovação e ciclo de vida diferentes do processo a que pertencem. Quanto às ontologias, desde o início da década de 90 (ALMEIDA, 2006, p. 107) têm ganhado notoriedade, tanto no contexto da Ciência da Informação, no contexto da Ciência da Computação e até mesmo no campo da Medicina.

A partir do que fora apresentado, será possível propor, por exemplo, a atribuição de um fluxo de gestão de conteúdo dos documentos e informações clínicas - sejam estas relativas ao evento de atendimento, ao âmbito de pesquisa ou administrativo. Tal conteúdo, a partir da representação das entidades reais e dos relacionamentos lógicos com seus respectivos universais correspondente, possibilitará agregar funcionalidades ao contexto.

Pode-se ainda pensar em vincular em um ambiente tecnológico as informações pertinentes ao paciente, seus exames, imagens, históricos e diagnósticos, incluindo as informações administrativas e relativas ao faturamento por meio de integrações sistêmicas que garantam a integridade dos significados. Desse modo, por meio da fundamentação ontológica de processos aplicada ao contexto clínico, será possível evidenciar que a prática da gestão de documentos e informações otimiza o uso dos recursos informacionais tecnológicos envolvidos nos processos de negócios. 
Teoricamente, o resultado que esse artigo apresenta consiste no avanço do entendimento dos processos em diferentes âmbitos de aplicação. Com o avançar da pesquisa, espera-se que seja possível identificar nos processos em modelagem as suas características essenciais e sua adequada categorização. Assim, por meio das notações, os processos apresentarão subsídios para a representação da realidade e das diferentes entidades processuais a eles relacionadas. Esses subsídios poderão se configurar como uma metodologia de referência para a comparação de processos e validação do modelo.

Fundamentam-se e contemplam-se com os desdobramentos deste trabalho, diferentes tipos de resultados de carácter pragmático que, embasados por consolidada literatura, constituirão novas premissas para o avanço da pesquisa das autoras. As mesmas têm buscado unir o conhecimento acadêmico e sua respectiva aplicação prática por meio de seus projetos desenvolvidos no mercado, valorizando os diferenciais conhecimentos do profissional da informação.

\section{REFERÊNCIAS}

AAGESEN, G.; KROGSTIE, J. Análise e estrutura de processos de negócios utilizando BPMN. In: BROCKE, J. V.; ROSEMANN, M. Manual de BPM: gestão de processo de negócio. Porto Alegre: Bookman, 2013.

AAGESEN, G.; KROGSTIE, J. BPM 2.0 for Modeling Business Process. In: Brocke, J. V.; Rosemann, M. Handbook on Business Process Management. 2. ed. Berlim/Heidelberg/Nova York: Springer-Verlag, 2015.

ABNT ASSOCIAÇÃO BRASILEIRA DE NORMAS TÉCNICAS. NBR ISO 9000:2015. Sistema de gestão da qualidade fundamentos e vocabulário, 2015

AGANETTE, E. C. Representação do Conhecimento Biomédico: Uma Investigação Sobre a Teoria Da Terminologia e a Teoria da Ontologia Aplicada no Domínio do Sangue Humano. 2015. Tese (Doutorado em Ciência da Informação) Escola de Ciência da Informação, Universidade Federal de Minas Gerais, Belo Horizonte, 2015.

AGANETTE, E. C.; TEIXEIRA, L.M.D.; AGANETTE, K. J. P. Modelagem de processos em empresa do setor de saúde pública: I-CISMEP, um relato de caso. Ágora, v. 28, n. 56, 2018.

ALMEIDA, M. B. Essencialismo. Belo Horizonte: PPGGOC-ECI/UFMG, 2014. 
ALMEIDA, M. B. Princípios ontológicos da organização da informação. Belo Horizonte: PPGGOC/UFMG, 2016. (Material didático)

ALMEIDA, M. B. Revisiting ontologies: a necessary clarification. Journal of the American Society of Information Science and Technology, v. 64, n. 8. p. 1682-1693, ago., 2013.

ALMEIDA, M. B. Um modelo baseado em ontologias para representação da memória organizacional. 2006. Tese (Doutorado em Ciência da Informação) Escola de Ciência da Informação, Universidade Federal de Minas Gerais, Belo Horizonte, 2006.

ALVARENGA NETO, R. C. D. Gestão do conhecimento em organizações: proposta de mapeamento conceitual integrativo. São Paulo: Saraiva, 2008.

BPMP. Guia para o Gerenciamento de Processos de Negócio: corpo comum de conhecimento (BPM CBOK®) versão 2.0 - terceira liberação em português. Chicago: ABPMP, 2009.

BRAUM, R.; BURWITZ, M.; SCHLIETER, H.; BENEDICT, M. Clinical processes from various angles: amplifying BPMN for integrated hospital management. In: INTERNATIONAL CONFERENCE ON BIOINFORMATICS AND BIOMEDICINE - BIBM, 2015, Washington, DC, USA. Anais [...]. Washington, DC, USA, 2015. Disponível em: https://ieeexplore.ieee.org/document/7359794. Acesso em: 17 nov. 2016

CAMPOS, M. L. A. Modelização de domínios de conhecimento: uma investigação de princípios fundamentais. Ciência da Informação, Brasília, v. 33, n. 1, p. 22-32, 2004.

CAMPOS, M. L. A.; CAMPOS, L. M. A Organização do conhecimento e suas teorias de representação: a ontologia de fundamentação como um modelo teórico para a representação de domínios. In: ENCONTRO NACIONAL DE PESQUISA EM CIÊNCIA DA INFORMAÇÃO, 13., 2012, Rio de Janeiro. Anais [...]. Rio de Janeiro: Fiocruz, 2012.

CHEHUEN NETO, J. M.; VALE, V. C. de S.; MAGALHÃES, G. G. R. Percepção médica quanto aos protocolos clínicos. HU Revista. v. 35, n. 3, Juiz de Fora, p. 159-166, jul./set.

CRUZ, T. Sistemas, métodos e processos: administrando organizações por meio de processos de negócios. Rio de Janeiro: Atlas, 2015.

DEMO, P. Pesquisa e construção do conhecimento: metodologia científica no caminho de Habermas. Rio de Janeiro: Tempo Brasileiro, 1994.

FNQ FUNDAÇÃO NACIONAL DA QUALIDADE. Introdução ao modelo de excelência da gestão. São Paulo: Fundação Nacional da Qualidade, 2007. 
FRANCELIN, M. M.; PELLEGATTI, C. Filosofia da informação: reflexos e reflexões. Transinformação, Campinas, v. 16, n. 2, p. 123-132, maio/ago. 2004.

GARVIN, D.A. The process of organization and management. Sloan Management Review, n.4, v. 39, p. 33-50, 1998.

GRENON, P.; SMITH, B., GOLDBERGH, L. Biodynamic Ontology: applying BFO in the biomedical domain. In: PISANELLI, D. M. Ontologies in Medicine. IOS Press, 2004.

GRUBER, T. R. Towards principles for the design of ontologies used for knowledge sharing. Int. J. Hum.-Comput. Stud., Palo Alto, CA, v. 43, p. 907928, 1993.

GRUNINGER, M.; PINTO, J. A. A theory of complex actions for Enterprise Modelling. In: BOUTILIER, C., GOLDSZMIDT, M. (org). Symposium Extending Theories of action: formal theory and practical applications. Stanford, California: AAAI Press, 1995.

GUIZZARDI, G., FALBO, R. A., GUIZZARDI, R. S. S. A importância de ontologias de fundamentação para a engenharia de ontologias de domínio: o caso do domínio de processos de software. IEEE Transactions Latin America, v. 6, p. 244-251, 2008.

GUIZZARDI, G.; WAGNER, G. A unified foundational ontology and some applications of it in business modeling. In: Conference on Advanced Information Systems Engineering, 16. 2004. Riga, Latvia. Anais [...]. Riga, Latvia: 2004. Disponível em: http://ftp.informatik.rwth-aachen.de/Publications/CEUR-WS/Vol125/paper2.pdf. Acesso em: 26 mar. 2017.

GUIZZARDI, G.; WAGNER, G. Some applications of a Unified Foundational Ontology in business modeling. In: GREEN, P; ROSEMANN, M. Business Systems analysis with ontologies. Hershey, PA: Idea Group, 2005.

GUIZZARDI, G.; WAGNER, G. Towards an ontological foundation of discrete event simulation. In: Proceedings of the 2010 Winter Simulation Conference, 2010. Baltimore, MD, USA. Anais [...]. Baltimore, MD, USA, 2010. Disponível em: http://www.informs-sim.org/wsc10papers/059.pdf. Acesso em: 26 mar. 2017.

HALLER, A.; GAALOUL, W.; MARMOLOWSKI, M. Towards an XPDL compliant process ontology. In: IEEE CONGRESS ON SERVICES, 1., 2008. Washington, DC, EUA. Anais [...]. Washington, DC, EUA: IEEE Computer Society, 2008. HAMMER, M.; CHAMPY, J. Reengineering the corporation. New York: Harper Business, 1994. 
HARRINGTON, H. J.; ESSELING, E. K. C.; NIMWEGEN, H. V. Business process improvement workbook: documentation, analysis, design and management of business process improvement. New York: McGraw Hill, 1997.

HENNING, B., Occurrents. In: MUNN, K.; SMITH, B. Applied Ontology: an introduction. Frankfurt: Ontos Verlag, 2008. Disponível em: http://ontology.buffalo.edu/AppliedOntology.pdf. Acesso em: 21 set. 2016.

KOBASHI, N. Y., TÁLAMO, M. de F. G. M. Informação: fenômeno e objeto de estudo da sociedade contemporânea. Transinformação, Campinas, v. 15, n. 3, p. 7-21, set./dez. 2003.

KROGSTIE, J.; DALBERG, V.; JENSEN, S. M. Process modeling value framework, enterprise information systems. In: MANOLOPOULOS, Y.; FILIPE, J.; CONSTANTOPOULOS, P.; CORDEIRO, J. (org.) Lecture notes in business information processing. Heidelberg: Springer-Verlag, 2008.

LWOFF, A. O conceito de informação na biologia molecular. In: ROYAUMONT, C. de (org.). $O$ conceito de informação na ciência contemporânea. Rio de Janeiro: Paz e Terra, 1970.

MARTINHO, R.; RIJO, R.; NUNES, A. Complexity analysis of a business process automation: case study on a healthcare organization. Procedia Computer Science, v. 64, 2015, p. 1226-1231. Disponível em: http://wwwsciencedirect-com.ez27.periodicos.capes.gov.br/science/article/pii/S1877050915026459. Acesso em: 23 nov. 2016

MAYR, E. The Growth of Biological Thought. Cambridge: Harvard University, 1982.

MENDES, R. D. Inteligência artificial; sistemas especialistas no gerenciamento da informação. Ciência da Informação, v. 26, n. 1, p. 39-45, 1998. Disponível em: http:/www.ibict.br/cienciadainformacao/viewarticle.php?id=458\&layout=abstract. Acesso: 10 de agosto de 2012.

MENDONÇA, F. M. Ontoforinfoscience: metodologia para construção de ontologias pelos cientistas da informação - uma aplicação prática no desenvolvimento da ontologia sobre componentes do sangue humano (HEMONTO). 2015. Tese (Doutorado). Programa de Pós Graduação em Ciência da Informação, UFMG, Belo Horizonte, 2015.

MOURELATOS, A. P. D. Events, process, and states. Linguistics and Philosophy, n. 2, p. 415-434, 1978. Disponível em: http://anon.cs.rochester.edu/u/james/Papers/mourelatos78.pdf. Acesso em: 16 fev. 2017.

OMG. BPMN 2.0 Specification: business process model and notation (BPMN) - version 2.0, technical reports. [S. I.: s. n.], 2011. Disponível em: http://www.omg.org/spec/BPMN/2.0/examples/PDF. Acesso em: 01 dez. 2016. 
PINHEIRO, L. V. R. Campo interdisciplinar da ciência da informação: fronteiras remotas e recentes. In: PINHEIRO, L. V. R. (org.). Ciência da informação, ciências sociais e interdisciplinaridade. Brasília; Rio de Janeiro: IBICT, 1999.

RECKER, J.; INDULSKA, M.; ROSEMANN, M.; GREEN, P. How good is BPMN really? Insights from theory and practice. In: EUROPEAN CONFERENCE ON INFORMATION SYSTEMS (ECIS), 2006. Gotemburgo, Suécia. Anais [...]. Gotemburgo, Suécia: AIS Electronic Library (AISeL), 2006. Disponível em: http://aisel.aisnet.org/cgi/viewcontent.cgi?article=1075\&context=ecis2006. Acesso em: 03 nov. 2016

RECTOR, A. L. Clinical terminology: why is it so hard? Methods of Information in Medicine, v. 38, n. 4, p. 239-252, 1999.

ROCHA, L. B. L da. Ontologias de notícias: um modelo para classificação do conteúdo dos jornais on-line brasileiros, segundo a lógica da semântica web. 2012. Dissertação (Mestrado). Programa de Pós-graduação em Design. UERJ, Rio de Janeiro, 2012. Disponível em: http://aflux.in/files/masters_thesis.pdf. Acesso em: 13 jun. 2018.

ROJO, M.G.; DANIEL, C.; SCHRADER, T. Standardization efforts of digital pathology in Europe. Analytical Cellular Pathology, n. 35, p. 19-23, 2012. Disponível em: https://www.hindawi.com/journals/acp/2012/858573/abs/. Acesso em: 27 dez. 2016

SCHEUERLEIN, H.; RAUCHFUSS, F.; DITTMAR, Y.; MOLLE, R.; LEHMANN, T.; PIENKOS, N.; SETTMACHER, U. New methods for clinical pathways Business Process Modeling Notation (BPMN) and Tangible Business Process Modeling (t.BPM). Langenbecks Arch Surg, n. 397, p. 755-761, 2012. Disponível em: http://link-springercom.ez27.periodicos.capes.gov.br/article/10.1007\%2Fs00423-012-0914-z. Acesso em: 20 dez. 2016

SHITKOWA, M.; TARATUKHIN, V.; BECKER, J. Towards a methodology and a tool for modeling clinical pathways. Procedia Computer Science, v. 63, p. 205212, 2015. Disponível em: http://www-sciencedirectcom.ez27.periodicos.capes.gov.br/science/article/pii/S1877050915024709. Acesso em: 04 jan. 2017.

SIMEONE, D.; KALAY, Y. E.; SCHAUMANN, D.; HONG, S. W. Modelling and simulating use processes in buildings. In: ECAADE COMPUTATION AND PERFORMANCE, 31., 2013, Holanda. Anais [...]. Holanda, 2013. Disponível em: http://papers.cumincad.org/data/works/att/ecaade2013_165.content.pdf. Acesso em: 16 dez. 2016.

SMITH, B. Beyond concepts: ontology as reality representation. [S. I.: s. n.], 2004. Disponível em: http://ontology.buffalo.edu/bfo/BeyondConcepts.pdf. Acesso em: 17 jun. 2018. 
SMITH, B. Ontology and informations systems. [S. I.: s. n.], 2003. Disponível em: http://ontology.buffalo.edu/ontology(PIC).pdf. Acesso em: 22 jun. 2012.

SMITH, B., ALMEIDA, M., BONA, J., BROCHHAUSEN, M., CEUSTERS, W., COURTOT, M., DIPERT, R., GOLDFAIN, A., GRENON, P., HASFNGS, J., HOGAN, W., JACUZZO, L., JOHANSSON, I., MUNGALL, C., NATALE, D., NEUHAUS, F., ROVEGO, A. P. R., RUGENBERG, A., RESSLER, M., AND SCHULZ, S. Basic Formal Ontology 2.0: draft specification and user's guide, 2012. Disponível em: http://bfo.googlecode.com/svn/trunk/docs/bfo2reference/BFO2-Reference.docx. Acesso em: 18 de junho de 2018.

STOUT, R. Processes. Philosophy, v. 72, p. 19-27, 1997.

TEIXEIRA, L. M. D.; AGANETTE, E. C. Ontologias no suporte à caracterização de processos: uma proposta de estudo. In: CONFERÊNCIAS IBERO-

AMERICANAS WWW/INTERNET E COMPUTAÇÃO APLICADA 2016, Lisboa, Portugal. Anais [...]. Lisboa, Portugal, 2016.

TEIXEIRA, L. M. D.; AGANETTE, E. C. Os processos de negócios, a gestão de documentos e os fluxos documentais: algumas perspectivas e relações. Rev.

Digit. Bibliotecon. Cienc. Inf., Campinas, v. 16, n. 3, p. 427-439, set./dez., 2018.

USCHOLD, M.; KING, M.; MORALEE, S.; ZORGIOS, Y. The enterprise ontology. AIAl: The University of Edinburgh, 1997.

VENDLER, Z. Linguistics in Philosophy. Ithaca: Cornell University Press, 1967.

VERNADAT, F. B. Enterprise modeling and integration: principles and applications. London, UK: Chapman and Hall, 1996.

\title{
FROM ONTOLOGICAL FOUNDATION TO THE REPRESENTATION OF CLINICAL PROCESSES: A THEORETICAL APPROACH
}

\begin{abstract}
Introduction: Managing issues related to the organization of information independent of the knowledge area is an arduous and costly task as it requires specific knowledge, appropriate methodologies and appropriate support tools. In this context, ontologies and process modeling have stood out as solutions for managing such problems, allied, as an alternative to achieve interoperability between systems. Objective: In view of this, the present article seeks to present a philosophical, ontological and process management rationale, as well as to show that the respective foundations are capable of supporting the need for improvements in business process modeling. Methodology: For this, we present a theoretical contextualization about processes in the philosophical scope, in corporate management, in domain ontologies and finally, contextualizes the theoretical body in clinical process management. Results: As a result, the article highlights the
\end{abstract}


progress of the understanding of the processes in different areas of application. As the research progresses, it is expected that it will be possible to identify in the modeling processes their essential characteristics and their proper categorization. Thus, through the notations, the processes will present subsidies for the representation of reality and the different procedural entities related to them. These subsidies can be configured as a reference methodology for process comparison and model validation. Conclusions: Clinical processes, the final objective of ongoing research, are developed in the health context and refer to routine in clinics, outpatient clinics, hospitals or even administrative activities. These processes are characterized by life-care involvement, where time is precious for decision making. It is expected that the developments provided by this study will be used as a theoretical foundation for the use of process-oriented ontologies in the clinical environment, contributing to their development in the field of Information Science.

Descriptors: Ontologies. Business Process Management. Process Modeling. Information Representation.

\title{
DE LA BASE ONTOLÓGICA A LA REPRESENTACIÓN DEL PROCESO CLÍNICO: UN ENFOQUE TEÓRICO
}

\begin{abstract}
RESUMEN
Introducción: la gestión de problemas relacionados con la organización de la información independientemente del área de conocimiento es una tarea ardua y costosa, ya que requiere un conocimiento específico, metodologías apropiadas y herramientas de apoyo adecuadas. En este contexto, las ontologías y el modelado de procesos se han destacado como soluciones para gestionar tales problemas, aliados, como una alternativa para lograr la interoperabilidad entre sistemas. Objetivo: en vista de esto, el presente artículo busca presentar una justificación filosófica, ontológica y de gestión de procesos, así como mostrar que las bases respectivas son capaces de respaldar la necesidad de mejoras en el modelado de procesos de negocios. Metodología: para ello, presentamos una contextualización teórica sobre procesos en el ámbito filosófico, en la gestión corporativa, en ontologías de dominio y finalmente, contextualizamos el cuerpo teórico en la gestión de procesos clínicos. Resultados: Como resultado, el artículo destaca el progreso de la comprensión de los procesos en diferentes áreas de aplicación. A medida que avanza la investigación, se espera que sea posible identificar en los procesos de modelado sus características esenciales y su categorización adecuada. Así, a través de las anotaciones, los procesos presentarán subsidios para la representación de la realidad y las diferentes entidades procesales relacionadas con ellos. Estos subsidios se pueden configurar como una metodología de referencia para la comparación de procesos y la validación de modelos. Conclusiones: Los procesos clínicos, el objetivo final de la investigación en curso, se desarrollan en el contexto de la salud y se refieren a la rutina en clínicas, clínicas ambulatorias, hospitales o incluso actividades administrativas. Estos procesos se caracterizan por la participación en el cuidado de la vida, donde el tiempo es precioso para la toma de decisiones. Se espera que los desarrollos proporcionados por este estudio se utilicen como una base teórica para el uso de ontologías orientadas a procesos en el entorno clínico, contribuyendo a su desarrollo en el campo de las Ciencias de la Información.
\end{abstract}

Descriptores: Ontologías. Gestión de Procesos de Negocios. Modelado de Procesos. Representación de la Información. 